\title{
Ultra-Sonography Guided Fine Needle Aspiration Cytology for the Diagnosis of Intra-Abdominal Lesions: An Institutional Study
}

\author{
Dr. Deepika Hemrajani ${ }^{1}$, Dr. Palak Upadhyay ${ }^{2 *}$ \\ ${ }^{1}$ Associate Professor, Department of Pathology, SMS Medical College, Jaipur, Rajasthan, India \\ ${ }^{2}$ Post Graduate Resident Doctor, Department of Pathology, SMS Medical College, Jaipur, Rajasthan, India
}

DOI: $10.36348 / \mathrm{sipm} .2020 . \mathrm{v} 05 \mathrm{i} 12.001$

| Received: 16.11.2020 | Accepted: 28.11.2020 | Published: 05.12.2020

*Corresponding author: Dr. Palak Upadhyay

\section{Abstract}

Guided FNAC as become a clinching investigation for intra-abdominal lesions be it palpable or non-palpable. FNAC as superseded the need for biopsy as it involves a complex setup for laparotomy procedure and very small and deep-seated lesions cannot be approached by biopsy. This study included all palpable and non-palpable lesions in different abdominal organs presented in various departments of Sawai Man Singh Hospital, Jaipur. Relevant investigations were taken by the patients before posting them for procedure including prothrombin time and contraindications considered like haemangiomas, bleeding tendencies. The aspirates were stained with $\mathrm{H}$ \&E stain and MGG stain and special stains applied when required. A total of 1220 cases were received in the duration of one year. A female preponderance was noted with M: F (1:1.32). The age range was from 11-month-old to 98-year-old patients. Maximum cases were in the age group of 51-60 years (219 cases). Liver was the most common site (45\%) followed by gall bladder (31.3\%) and then pancreas $(6.55 \%)$. Majority of lesions were malignant $(55.8 \%)$ followed by benign (15\%). Malignant lesions were noted more in females whereas male population had more of benign and inflammatory lesions. The rate of malignant lesions increased as the age increased. In liver most common malignancies were metastatic lesions whereas in gall bladder, primary lesions were more common. FNAC has a very high sensitivity which makes it a first line investigation in evaluation of abdominal lesions. It has both diagnostic and prognostic value and enables a surgeon for treatment modalities.

Keywords: USG-Guided, FNAC, Intra-abdominal lesions, Malignancies, Hepatobiliary, Cell block.

Copyright $\odot 2020$ The Author(s): This is an open-access article distributed under the terms of the Creative Commons Attribution 4.0 International License (CC BY-NC 4.0) which permits unrestricted use, distribution, and reproduction in any medium for non-commercial use provided the original author and source are credited.

\section{INTRODUCTION}

Intra-abdominal lesions are a perfect paradigm for a surgeon's clinical dilemma as these being the most commonly encountered complain; carry a wide range of spectrum from being benign to inflammatory to various grades of malignancy. In the present era where needle is preceding the scalpel, guided FNAC of abdominal lesions is of utmost importance not only diagnostically but also prognostically as early and accurate treatment can cure the disease thereby reducing the morbidity and mortality rates and improving the quality of life of patients.

The diagnosis of the nature of intra-abdominal lesions involves a wide array of investigations involving radiological, cytological and serological spectrums. The introduction of modern diagnostic imaging techniques such as ultrasonography (USG), computed tomography (CT SCAN) and magnetic resonance imaging (MRI) has enabled the detection of the exact location of intra-abdominal lesions. Furthermore, CT scans and MRI are as well very efficient in staging the tumours in cases of malignancy. Only imaging per se cannot exactly categorise the nature of the lesions as it is not $100 \%$ efficient and hence the use of cytological study of these lesions carries the utmost importance.

Nowadays Endoscopic ultrasound (EUS) guided needling is increasingly being used for the lesions accessible through Tran's gastric approach [13]. Duct brush cytology and endoscopic retrograde cholangiopancreatography (ERCP) has gained wide utility in evaluation of extrahepatic biliary tract and large pancreatic duct lesions [4].

Guided FNAC is the earliest, readily acceptable, easily approachable, time saving, easiest and accurate procedure for the diagnosis of any intraabdominal lesion. Usually the abdominal masses require biopsies by laparotomy procedures which are 
not possible in all cases due to very small lesions or inaccessible sites. Such obstacles are easily overcome by the use of guided FNACs which render a cytohistologic diagnosis in space occupying lesions of the abdomen [5]. The basic principle of USG guided FNAC is that it converts a complicated three-dimensional problem of hitting a small lesion into a much easier two-dimensional imaging technique[5].

It also offers the advantage of non-exposure to ionizing radiations and it can provide guidance in multiple planes such as transverse, oblique and longitudinal. The best advantage, however, remains the real time visualization of the needle tip as it passes through the tissue planes into the target tissue. Very small lesions, even up to $1 \mathrm{~cm}$ can be accessed by guided FNAC procedure with accuracy, the disadvantage that is carried in cases of biopsies [5].

The whole procedure is a team work of both radiologist and the pathologist as the presence of pathologist ensures the proper cellular content on the smears, the clinical correlation required, and suggestion of any additional biopsies and also the application of special procedures such as special stains or sending the samples for culture in cases of abscesses or tuberculosis.

In recent times the preparation of cell blocks from the aspirate is gaining wide popularity and can be extremely useful diagnostically in cases of lymph nodal diseases like lymphomas where the biopsy from that lymph node cannot be accessed. The preparation of cell blocks and application of immunohistochemistry (IHC) on such cases is equivalent to the biopsies obtained.

The present study was undertaken to critically analyse the utility of guided FNAC as the pre-operative diagnostic procedure in management of intra-abdominal lesions and to study the spectrum of these lesions depending on the age, gender, site, type of lesion whether benign, inflammatory or malignant. In cases of malignancy the exact cell type and whether primary or metastatic.

\section{MATERIALS AND METHODS}

The study involved 1220 cases diagnosed clinically or radiologically as abdomino-pelvic masses, which were referred to the Department of Pathology, Sawai Man Singh Medical College, Jaipur, and Rajasthan during the period of one year from January 2019 to December 2019.

Majority of patients presented with mass per abdomen and/or pain in the abdomen. Detailed clinical history including the duration of complains, associated symptoms, reports of previous investigations including coagulation profiles were obtained.
Before posting the patients for the procedure, they were explained the procedure and the contraindications were considered like prolonged prothrombin time, haemangiomas, echinococcosis and uncooperative patients. The patients were examined ultrasonographically for the origin of the mass and its anatomic relations with adjacent organs.

A real time USG guided FNAC was taken in the Department of Radiology while taking proper aseptic precautions by the shortest route to the site. A $10 \mathrm{ml}$ disposable plastic syringe and a 22-gauge needle were used. The aspirates were taken on 5-6 glass slides. Those that were immediately fixed in $95 \%$ alcohol were stained by Haematoxylin and Eosin stain and those that were dried were stained by May Grunwald Giemsa stain. Special stains were applied wherever required.

Only those smears which had adequate cellularity were taken for analysis. Patients with masses in intra-abdominal organs like liver, gall bladder, intestine, soft tissues, ovaries, pancreas, lymph nodes, mesentery, retroperitoneum, kidney and adrenals and patients those were known cases of a malignancy were taken for metastatic site evaluation.

Parietal swellings arising from skin, uterus, cervix, prostate and bones, patients with prolonged PT, haemorrhagic tendencies, haemangiomas and uncooperative patients and aspirates with inadequate cellularity were excluded.

\section{RESULTS}

A total of 1220 cases were included in this study with detailed clinical history, serological markers and other lab investigations. Majority of patients were females with a male female ratio of 1:1.32 as shown in table no 1 . The age range was from an 11 months old female child to 98-year-old male. The most common site was liver $(45 \%)$ followed by gall bladder $(31.3 \%)$ and then pancreas $(6.55 \%)$ as shown in table no.2.Majority of cases were malignant (55.8\%) followed by benign cases (15\%) as shown in table no.3.

Maximum cases were malignant lesions with predominant age group of 51-60 years (219 cases) with females having a greater number of malignant cases (124) than males (95) followed by the age group of 6170 years (190 cases). Maximum number of benign cases were seen in the age group of 11-20 years (60 cases) followed by the age group of 51-60 years (44 cases). Inflammatory lesions were more common in males (54) than females (22). Similar findings were observed with the benign lesions i.e., males (131) more than females (52) as shown in table no.4.

The most common organ involved in malignancy was liver with secondaries $(66 \%)$ being more common than hepatocellular carcinoma (34\%) per se. The various types of secondaries were 
adenocarcinomas, adenosquamous carcinomas, haematolymphoid malignancies, adenoid cystic and small cell tumours as shown in table no.3.

The second most common involved organ in malignancy was gall bladder with primary carcinomas of gall bladder being more common than secondaries in it. The types of secondaries were adenocarcinoma, adenosquamous carcinoma, signet ring cell carcinoma, mucinous carcinomas and gastrointestinal stromal tumours.

Pancreas and the lymph nodes including inguinal, retroperitoneal and iliac lymph nodes had only $5-10 \%$ primaries and $90-95 \%$ secondaries.

Table-1: Sex wise distribution of lesions

\begin{tabular}{|l|l|l|l|l|}
\hline LESION TYPE & MALE & TOTAL \% & FEMALE & TOTAL\% \\
\hline Inflammatory & 54 & $8.95 \%$ & 22 & $3.57 \%$ \\
\hline Benign & 131 & $21.68 \%$ & 52 & $8.45 \%$ \\
\hline Malignant & 299 & $49.50 \%$ & 382 & $62.01 \%$ \\
\hline Unsatisfactory & 120 & $19.87 \%$ & 160 & $25.97 \%$ \\
\hline Total & $\mathbf{6 0 4}$ & $\mathbf{1 0 0}$ & $\mathbf{6 1 6}$ & $\mathbf{1 0 0}$ \\
\hline
\end{tabular}

Table-2: Organ wise distribution of cases

\begin{tabular}{|l|l|l|}
\hline ORGAN & NUMBER OF CASES & TOTAL \% \\
\hline Liver & 549 & $45 \%$ \\
\hline Gall bladder & 382 & $31.33 \%$ \\
\hline Pancreas & 80 & $6.57 \%$ \\
\hline Spleen & 10 & $0.82 \%$ \\
\hline Renal & 25 & $2.04 \%$ \\
\hline Adrenal & 04 & $0.32 \%$ \\
\hline Omentum & 25 & $2.04 \%$ \\
\hline Retroperitoneum & 10 & $0.82 \%$ \\
\hline Iliac lymph node & 19 & $1.56 \%$ \\
\hline Inguinal lymph node & 72 & $5.90 \%$ \\
\hline Ovary & 44 & $3.60 \%$ \\
\hline Total & $\mathbf{1 2 2 0}$ & $\mathbf{1 0 0}$ \\
\hline
\end{tabular}

Table-3: Distribution of malignant cases

\begin{tabular}{|l|l|l|}
\hline TYPE OF LESION & NUMBER OF CASES & TOTAL \% \\
\hline Adenocarcinoma & 394 & $57.86 \%$ \\
\hline Hepatocellular carcinoma & 60 & $8.82 \%$ \\
\hline Cholangiocarcinoma & 167 & $24.52 \%$ \\
\hline Renal cell carcinoma & 10 & $1.46 \%$ \\
\hline Adenosquamous & 10 & $1.46 \%$ \\
\hline Neuroendocrine tumours & 08 & $1.17 \%$ \\
\hline Non-Hodgkin's lymphoma & 07 & $1.02 \%$ \\
\hline Squamous cell carcinoma & 07 & $1.02 \%$ \\
\hline Small cell carcinoma & 03 & $0.44 \%$ \\
\hline Round cell neoplasm & 03 & $0.44 \%$ \\
\hline Malignant melanoma & 03 & $0.44 \%$ \\
\hline GIST & 02 & $0.30 \%$ \\
\hline Pseudopapillary neoplasm & 02 & $0.30 \%$ \\
\hline Adrenocortical neoplasm & 01 & $0.15 \%$ \\
\hline Germ cell tumour & 01 & $0.15 \%$ \\
\hline Paraganglioma & 01 & $0.15 \%$ \\
\hline Hepatoblastoma & 01 & $0.15 \%$ \\
\hline Anaplastic carcinoma & 01 & $0.15 \%$ \\
\hline Total & 681 & 100 \\
\hline
\end{tabular}


Table-4: Age wise, sex wise and type wise lesion categorisation

\begin{tabular}{|c|c|c|c|c|c|c|c|c|}
\hline \multirow[t]{2}{*}{ AGE } & \multicolumn{2}{|c|}{ INFLAMMATORY } & \multicolumn{2}{|c|}{ BENIGN } & \multicolumn{2}{|c|}{ MALIGNANT } & \multicolumn{2}{|c|}{ UNSATISFACTORY } \\
\hline & $\mathrm{M}$ & $\mathrm{F}$ & $\mathrm{M}$ & $\mathrm{F}$ & M & $\mathrm{F}$ & $\mathrm{M}$ & $\mathrm{F}$ \\
\hline$<1$ year & & 1 & & & & & & \\
\hline $1-10$ & 2 & 2 & & 1 & 1 & & 1 & 1 \\
\hline $11-20$ & 9 & 5 & 40 & 20 & 1 & 3 & 1 & 1 \\
\hline $21-30$ & 7 & 4 & 20 & & 4 & 8 & 10 & 20 \\
\hline $31-40$ & 10 & 2 & 21 & 10 & 15 & 35 & 20 & 33 \\
\hline $41-50$ & 10 & 2 & 10 & & 70 & 80 & 20 & 35 \\
\hline $51-60$ & 5 & 2 & 30 & 14 & 95 & 124 & 25 & 25 \\
\hline $61-70$ & 10 & 2 & 10 & 3 & 90 & 100 & 16 & 25 \\
\hline $71-80$ & 1 & 1 & & 2 & 20 & 25 & 26 & 20 \\
\hline $81-90$ & & 1 & & 2 & 3 & 6 & & \\
\hline 91-100 & & & & & & 1 & 1 & \\
\hline Total & 54 & 22 & 131 & 52 & 299 & 382 & 120 & 160 \\
\hline
\end{tabular}

Table-5: Comparison of nature of lesions with other studies

\begin{tabular}{|l|l|l|l|}
\hline Type of lesion & Inflammatory & Malignant & Unsatisfactory \\
\hline Aftab A et al. $[13]$ & $6 \%$ & $57.5 \%$ & $6.5 \%$ \\
\hline Shamshad et al. [4] & $30.5 \%$ & $57.5 \%$ & $6.5 \%$ \\
\hline Sidhalingreddy et al. $[10]$ & $10.2 \%$ & $60.3 \%$ & $6.5 \%$ \\
\hline Madhav N et al. [15] & $22.56 \%$ & $71.93 \%$ & $3.65 \%$ \\
\hline Present study 2020 & $6.22 \%$ & $55.81 \%$ & $22.95 \%$ \\
\hline
\end{tabular}

Table-6: Comparing most common malignant cell type with other studies

\begin{tabular}{|l|l|l|l|l|l|}
\hline Ref no & Study & $\begin{array}{l}\text { Adenocarcinoma } \\
\text { (No of cases) }\end{array}$ & $\begin{array}{l}\text { Total } \\
(\%)\end{array}$ & Hepatocellular carcinoma (no of cases) & $\begin{array}{l}\text { Total } \\
(\%)\end{array}$ \\
\hline 1 & Shamshad et al. $[4]$ & 12 & 60 & 8 & 40 \\
\hline 2 & Sidhalingreddy et al. $[10]$ & 39 & 26.3 & 34 & 23 \\
\hline 3 & AS Tuladhar et al. $[14]$ & 5 & 45.5 & 3 & 27.2 \\
\hline 4 & Kothari et al. $[19]$ & 37 & 64.91 & 10 & 14.93 \\
\hline 5 & Madhav N et al. $[15]$ & 69 & 58.47 & 34 & 33.05 \\
\hline 6 & Present study $(2020)$ & 394 & 57.86 & 60 & 8.82 \\
\hline
\end{tabular}

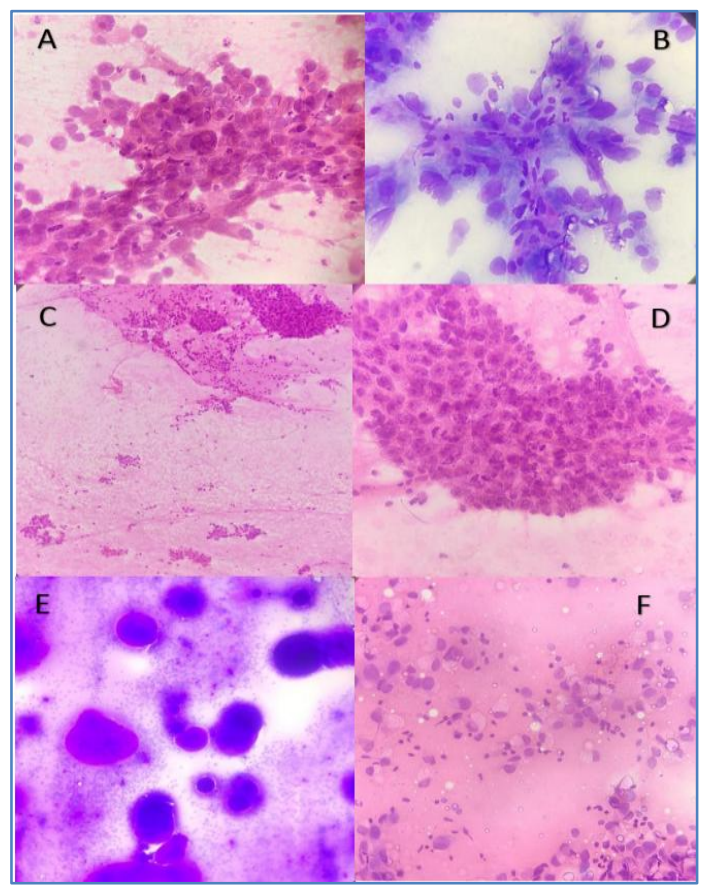

Fig-1: Photomicrographs of $H \& E$ and MGG stained smears of (A)High power view(400X) of Hepatocellular carcinoma showing polygonal cells with high N/C ratio,central round nuclei with prominent macronucleoli and marked nuclear atypia.(B)MGG stained high power view (400X)of same case showing transgressing of vessels.(C)Low power view(100X) of case of testicular tumour metastasising to liver showing Schiller Duval bodies in lower left corner.(D)High power view(400X) of same case showing marked nuclear atypia, high N/C ratio, prominent 1-2 nucleoli and brisk mitosis.(E)High power view(400X) MGG stained case of adenoid cystic carcinoma metastasising to liver showing hyaline spherical globules of varying sizes with adherent tumour cells and presence of hyaline stroma .(F) High power view $(400 \mathrm{X})$ of a case of signet ring cell carcinoma metastasising to gall bladder showing presence of intra and extra cellular mucin. intracellular mucin has displaced the nucleus to the periphery 


\section{DISCUSSION}

Guided FNAC has been a boon for the diagnosis of intra abdominal lesions as they are the most commonly encountered clinical manifestation in surgical practices. It has removed the cumbersome process of laparotomy for taking biopsies by a simple needle guided procedure which is easy, time saving, easily accessible and accurate. It is sometimes superior to a biopsy as it can be used for as small lesions as $1 \mathrm{~cm}$ as well as those lesions that are inaccessible by biopsy needles.

The initial target of FNAC was palpable masses like enlarged lymph nodes , breast lumps, thyroid but now FNAC of intra abdominal lesions is becoming the first line investigation[5-7]

The present study included 1220 cases of palpable as well as non palpable masses from various abdominal organs. Out of 1220 cases, 604 were males and 616 were females with a ratio of $1: 1.32$ which was in concordance with observations made by Shamshad et al. [8] and Joao Nobrega et al. [9] and Sidhalingreddy [10]. But the observations made in the studies by Zawar et al. [11] and Govind Krishna et al. [12] showed a male predominance.

Benign lesions were more commonly seen in patients below 40 years of age while the incidence of malignancy increased with age. The peak incidence of malignancy was between the age of 51-60 years of age which is comparable with the results obtained by Zawar et al. [11] and Shamshad et al. [8]. In our study the most common lesion was malignant. Other studies like Shamshad et al. [8] , Aftab A Khan [13] , Sidhalingreddy et al. [10] also had the similar findings as shown in table no 5 .

Liver was the most common site for FNAC in this study as shown in table 2 which is comparable to studies done by Sheihk et al. [6] and Adhikari et al. [14] and Madhav N et al. [15]. This is also the most common site for aspiration performed in the abdomen as also shown in studies by J Nobrega et al. [9] , Aftab A Khan et al. [13], Zawar et al. [11].

The second most common site in our study was gall bladder which is also in concordance with study by Reyaz et al. [16], Shamshad et al. [8], Adhikari et al. [14] and Madhav N et al. [15].

In present study adenocarcinoma was the most common malignancy noted in accordance with Zawar et al. [11]. The peak age of adenocarcinomas was 40-60 years in concordance with studies of Shamshad et al. [8] and Zawar et al. [11] as shown in table no 6.

The most common presenting complain was abdominal pain in right hypochondrium, loss of appetite, weight loss and/or abdominal lump, which is also similar to studies by Ali et al. [17] and Rassnia et al. [18] and Madhav N et al. [15]. Lesser cases also had history of jaundice and pruritis.

The unsatisfactory / acellular smears could be due to a number of factors like very deep location not reachable by the needle, high vascularity of the lesion, larger necrotic component or very small size of the lesion. Such factors can be considered before performing FNAC and it can improve the diagnostic yield.

FNAC is by far a very safe and accurate procedure though very few studies report complications like mild local pain, bleeding and tumour seeding of needle tract. Presence of pathologist is of utmost importance keeping in mind the cell content examination, proper clinical correlation, imaging findings and any additional stains or culture tests to be done.

\section{CONCLUSION}

In the present study $55.81 \%$ malignant lesions were diagnosed by a simple outpatient procedure with a good cost benefit ratio. The most common lesion was malignant hepatic lesion followed by gall bladder malignancies.

There were minimal complications with less time consumption and an easy, accessible procedure for the patient. It replaced a whole complicated process of laparotomy for biopsies which requires a special set up by a simple needle guided procedure. Also there could be many passes to obtain a proper yield as oppose to biopsies. Since it is USG guided there is proper anatomical location of the lesion with the current USG findings noted and any abnormality or infiltration in the adjacent organs can be noted too.

The most recent advantage is the development of cell block preparations by the aspirate. The cell block preparation allows the evaluation of ancillary studies such as immunohistochemistry (IHC) on the aspirate which is as good as biopsy.

FNAC is a highly sensitive, specific, accurate and cost effective diagnostic procedure for intra abdominal lesions with minimum complications. With the increasing use of cell blocks for IHC and flow cytometry for lymphomas, its sensitivity and accuracy has been found to be as good as biopsy.

\section{REFERENCES}

1. Thuluvath, P.J. (2007). EUS guided FNA could be another important tool for the early diagnosis of hepatocellular carcinoma. Gastrointestinal Endoscopy, 66,274-6.

2. Giovannini, M., Seitz, J.F., Monges, G., Perrier, H., Rabbia, I. (1995). Fine -needle aspiration cytology 
guided by endoscopic ultrasonography: Results in 141 patients. Endoscopy, 27,171-7.

3. Bentz, J.S., Kochman, M.L., Faigel, D.O., Ginsberg, G.G., Smith, D.B., Gupta, P.K. (1998). Endoscopic ultrasound-guided real time fine needle aspiration: Clinicopathologic features of 60 patients. Diagnostic Cytopathology, 18, 98-109.

4. Brugge, W.R., Lauwers, Y., Sahani, D., Fernandezdel, Castillo. C., Warshaw, A.L. (2004). Cystic neoplasms of the pancreas. New England Journal of Medicine, 351, 1218-26.

5. Hemlatha, A.L., Sumana, Sushma. S., Varna, I. (2013). Ultrasound guided FNAC of abdominalpelvic Masses-The Pathologists Perspective. Journal of Clinical and Diagnostic Research for Doctors, 7(2),273-77.

6. Sheikh, M., Sawhney, S., Dey, P., AI-Saeed, O., Behbehani, A. (2000). Deep seated thoracic and abdominal masses: Usefulness of ultrasound and computed tomography guidance in fine needle aspiration cytology diagnosis. Australasian Radiology, 44,155-60.

7. Wilson, Man-Shan., Tsui, Fung-yip. C., Yat -wing, Lee. (1998). Fine needle aspiration cytology of liver tumour. Annals of Diagnostic Pathology, 2; 79-93.

8. Ahmed, S., Akhtar, K., Akhtar, S. (2006). Ultrasound guided fine meddle aspiration biopsy of abdominal masses. Jammu \& Kashmir Science, 8(4), 200-4.

9. Nobrega, J., Santos, G. (1994). Aspiration cytology with fine needle in the abdomen, retroperitoneum and pelvic cavity: a seven-year experience of the Portuguese institute of oncology. European Journal of Surgical Oncology, 20, 37-42.

10. Sidhalingreddy, Sainath, K.A. (2011). Fine needle aspiration of intra-abdominal lesion. Journal of Clinical and Diagnostic Research, 5(3).
11. Zawar, M.P., Bolde, S., Shete, S.S. (2007). Correlative study of fine needle aspiration cytology and histology in intra-abdominal lumps. Singapore Medical Journal, 4.

12. Govind, Krishna, S.R., Ananthakrishanan, N., Narasimhan, R., Veliath, A.J. (1993). Accuracy of fine needle aspiration cytology of abdominal masses without radiological guidance. Indian Journal of Pathology and Microbiology, 36(4), 442-52.

13. Khan, A., Jan, G.M., Wani, N.A. (1996). Fine needle aspiration of Intra-abdominal masses for cytodiagnosis. Journal of Indian Medical Association, 94(5), 167-69.

14. Adhikari, R.C., Tuladhar, A., Shreshta, S., Sharma, S.K. (2010). Deep seated thoracic and abdominal lesions: usefulness of ultrasound guided fine needle aspiration cytology, a 3-year experience. Nepal Medical College Journal, 12, 20-25.

15. Madhav, N., Meenai, F.J. (2019). Role of USG guided FNAC in intra-abdominal masses- A study at tertiary care hospital Bhopal. IP Archives of Cytology and Histopathology Research, JanuaryMarch, 4(1), 19-25.

16. Reyaz, T.A., Summyia, F., Isma, N., Nazia, B., Adil, S., Sameena, K. (2016). USG guided fine needle aspiration cytology of intra-abdominal and pelvic masses in Kashmir: A study at tertiary care hospital. International Journal of Medical Research and Health Sciences, Jan 1, 5(4), 169-75.

17. Ali, S.R., Jayabackthan, L., Rahim, S., Sharel, M.B., Prasad, K., Hegdekatte, N. (2015). Role of FNAC in the diagnosis of hepatic lesions. Muller Journal of Medical Sciences and Research, 6,12528.

18. Rasania, A., Pandey, C.L., Joshi, N. (2007). Evaluation of FNAC in diagnosis of hepatic lesions. Journal of Cytology, 24, 51-4. 